\title{
Anisotropic thermal magnetoresistance for an active control of radiative heat transfer
}

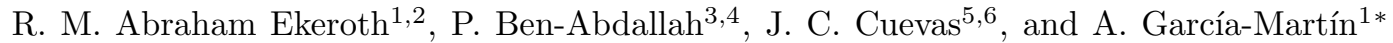 \\ ${ }^{1}$ IMN-Instituto de Micro y Nanotecnología (CNM-CSIC), \\ Isaac Newton 8, PTM, Tres Cantos, E-28760 Madrid, Spain \\ ${ }^{2}$ Instituto de Física Arroyo Seco, Universidad Nacional del Centro \\ de la Provincia de Buenos Aires, Pinto 399, 7000 Tandil, Argentina \\ ${ }^{3}$ Laboratoire Charles Fabry, UMR 8501, Institut d'Optique, CNRS, \\ Université Paris-Saclay, 2 Avenue Augustin Fresnel, 91127 Palaiseau Cedex, France \\ ${ }^{4}$ Université de Sherbrooke, Department of Mechanical Engineering, Sherbrooke, PQ J1K 2R1, Canada \\ ${ }^{5}$ Departamento de Física Teórica de la Materia Condensada and Condensed Matter Physics Center (IFIMAC), \\ Universidad Autónoma de Madrid, E-28049 Madrid, Spain and \\ ${ }^{6}$ Department of Physics, University of Konstanz, D-78457 Konstanz, Germany
}

(Dated: June 26, 2018)

\begin{abstract}
We predict a huge anisotropic thermal magnetoresistance (ATMR) in the near-field radiative heat transfer between magneto-optical particles when the direction of an external magnetic field is changed with respect to the heat current direction. We illustrate this effect with the case of two InSb spherical particles where we find that the ATMR amplitude can reach values of up to $800 \%$ for a magnetic field of $5 \mathrm{~T}$, which is many orders of magnitude larger than its spintronic analogue in electronic devices. This thermomagnetic effect could find broad applications in the fields of ultrafast thermal management as well as magnetic and thermal remote sensing.
\end{abstract}

In 1857 Thomson discovered that the resistivity of bulk ferromagnetic metals depends on the relative angle, $\theta$, between the electric current and the magnetization direction 11. This phenomenon, known as anisotropic magnetoresistance (AMR), plays nowadays a central role in the field of spintronics [2] and it is the basis of sensors for magnetic recording [3, 4]. The AMR originates from the anisotropy of electron scattering due to the spin-orbit interaction [4. In bulk samples the rotation of the magnetization leads to a relative change in the resistance that varies as $\cos ^{2} \theta$ with an amplitude on the order of $1 \%$, while it has been recently shown that this amplitude can be increased by an order of magnitude in atomic-scale ferromagnetic junctions [5]10. In this Letter we predict the existence of a thermal analogue of AMR in the context of radiative heat transfer between magneto-optical (MO) particles. In particular, we predict that, in the near-field regime, this effect can be several orders of magnitude larger than its spintronic counterpart.

In recent years, the field of thermal radiation has received a new impetus from the confirmation that the radiative heat transfer between two closely placed objects can greatly overcome the far-field limit set by the Stephan-Boltzmann law [11 24. This enhanced thermal radiation stems from the contribution of evanescent waves (photon tunneling) that dominate the near-field regime. At present, one of the central challenges in this field is to actively control the near-field radiative heat transfer (NFRHT). In this context, magneto-optical (MO) objects has been put forward as a promising avenue to control the NFRHT with an external magnetic field [25]. In the last two years, several thermomagnetic effects have been predicted such as a near-field thermal

\footnotetext{
* a.garcia.martin@csic.es
}

Hall effect [26], the existence of a persistent heat current 27, or a giant thermal magnetoresistance 28. However, most of the attention has been devoted to the role of the magnitude of the field. In this work, we show that the NFRHT between two MO particles can be efficiently controlled by changing the direction of the magnetic field, in the spirit of the AMR in spintronics. This phenomenon, which we term anisotropic thermal magnetoresistance (ATMR), stems from the anisotropy of the photon tunneling induced by the magnetic field. We discuss this effect through the analysis of the radiative heat exchange between two InSb particles, see Fig. 1, and show that the ATMR can reach amplitudes of $100 \%$ for fields on the order of $1 \mathrm{~T}$ and up to $1000 \%$ for a magnetic field of $6 \mathrm{~T}$. These values are several orders of magnitude larger than in standard spintronic devices $[3,4$.

For illustrative purposes, we consider here two identical spherical MO particles of radius $r$ embedded in vacuum and separated by a gap $d$, as sketched in Fig. 1. We assume that these particles are held at temperatures

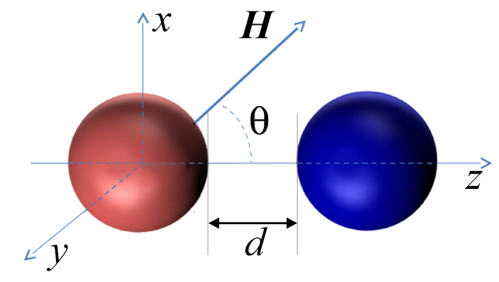

FIG. 1. (Color online) Sketch of the system with two identical magneto-optical particles of radius $r$ held at different temperatures and separated by a gap $d$. A magnetic field $\boldsymbol{H}$ lying in the $x z$-plane is applied in a direction forming an angle $\theta$ with the heat transport direction ( $z$-axis). 
$T+\Delta T$ and $T$ with $\Delta T \ll T$ (linear response regime) and they are subjected to a magnetic field $\boldsymbol{H}$ that forms an angle $\theta$ with the axis linking the two particles, see Fig. 1. To describe the radiative heat transfer between these two particles within the framework of fluctuational electrodynamics 29], we use the thermal discrete dipole approximation (TDDA) of Ref. [30. This method allows us to compute numerically the thermal radiative properties of MO particles of arbitrary size and shape by discretizing the objects in terms of point dipoles in the spirit of the DDA approach [31, 32. Within the TDDA approach, the radiative thermal conductance between two objects is given by the following Landauer-like formula

$$
G(H, \theta)=\int_{0}^{\infty} \frac{d \omega}{2 \pi} \frac{\partial \Theta(\omega, T)}{\partial T} \mathcal{T}(\omega, H, \theta),
$$

where $\Theta(\omega, T)=\hbar \omega /\left[\exp \left(\hbar \omega / k_{\mathrm{B}} T\right)-1\right]$ is the mean energy of an harmonic oscillator in thermal equilibrium at temperature $T$ and $\mathcal{T}(\omega, H, \theta)$ is the transmission coefficient that depends on the frequency $\omega$, the magnitude of the field $H$ and its direction. In general, this transmission coefficient has to be computed numerically with the TDDA approach and we refer to Ref. 30] for the technical details. To get some analytical insight, we shall also make use of the dipolar approximation in which, when the particles are small in comparison with the thermal wavelength, $\lambda_{\mathrm{Th}}=\hbar c /\left(k_{\mathrm{B}} T\right)$, they can be considered as single point dipoles. In this case, the transmission coefficient appearing in Eq. (1) adopts the form [30.

$$
\mathcal{T}(\omega, H, \theta)=4 k_{0}^{4} \operatorname{Tr}\left\{\hat{C} \hat{\chi} \hat{C}^{\dagger} \hat{\chi}\right\}
$$

where the susceptibility tensor $\hat{\chi}$ and $\hat{C}$ are $(3 \times 3)$ matrices given by

$$
\begin{aligned}
& \hat{\chi}=\frac{1}{2 i}\left(\hat{\alpha}-\hat{\alpha}^{\dagger}\right)-\frac{k_{0}^{3}}{6 \pi} \hat{\alpha}^{\dagger} \hat{\alpha}, \\
& \hat{C}=\left[\hat{1}-k_{0}^{4} \hat{\mathcal{G}} \hat{\alpha} \hat{\mathcal{G}} \hat{\alpha}\right]^{-1} \hat{\mathcal{G}} .
\end{aligned}
$$

Here, $k_{0}=\omega / c$ and $\hat{\alpha}$ is the polarizability tensor of the particles that is given by $\hat{\alpha}=\left[\hat{\alpha}_{0}^{-1}-i \hat{1} k_{0}^{3} /(6 \pi)\right]^{-1}$, where $\hat{\alpha}_{0}=3 V(\hat{\epsilon}-\hat{1})(\hat{\epsilon}+2 \hat{1})^{-1}$ is the quasistatic polarizability tensor [33]. Here, $V=(4 / 3) \pi r^{3}$ is the volumen of the particles and $\hat{\epsilon}$ is the corresponding permittivity tensor. Finally, $\hat{\mathcal{G}}$ is the dyadic Green tensor given by 34

$$
\begin{aligned}
\hat{\mathcal{G}}\left(\mathbf{r}_{1}, \mathbf{r}_{2}\right)= & \frac{e^{i k_{0} \rho}}{4 \pi \rho}\left[\left(1+\frac{i k_{0} \rho-1}{k_{0}^{2} \rho^{2}}\right) \hat{1}+\right. \\
& \left.\left(\frac{3-3 i k_{0} \rho-k_{0}^{2} \rho^{2}}{k_{0}^{2} \rho^{2}}\right) \frac{\boldsymbol{\rho} \otimes \boldsymbol{\rho}}{\rho^{2}}\right],
\end{aligned}
$$

where $\mathbf{r}_{1,2}$ are the positions of the point dipoles, $\boldsymbol{\rho}=$ $\mathbf{r}_{1}-\mathbf{r}_{2}, \rho=\left|\mathbf{r}_{1}-\mathbf{r}_{2}\right|$, and $\otimes$ denotes the exterior product.

As an example of a MO material we consider $n$-doped InSb, a polar semiconductor, that when subjected to an external magnetic field becomes MO. For an a magnetic field lying on the $x z$ plane and forming an angle $\theta$ with the $z$-axis, see Fig. 1] the permittivity tensor of $\mathrm{InSb}$ adopts the form 35 .

$\hat{\epsilon}=\left(\begin{array}{ccc}\epsilon_{1} \cos ^{2} \theta+\epsilon_{3} \sin ^{2} \theta & -i \epsilon_{2} \cos \theta & \frac{1}{2}\left(\epsilon_{1}-\epsilon_{3}\right) \sin (2 \theta) \\ i \epsilon_{2} \cos \theta & \epsilon_{1} & i \epsilon_{2} \sin \theta \\ \frac{1}{2}\left(\epsilon_{1}-\epsilon_{3}\right) \sin (2 \theta) & -i \epsilon_{2} \sin \theta & \epsilon_{1} \sin ^{2} \theta+\epsilon_{3} \cos ^{2} \theta\end{array}\right)$,

where

$$
\begin{aligned}
\epsilon_{1}(H) & =\epsilon_{\infty}\left(1+\frac{\omega_{L}^{2}-\omega_{T}^{2}}{\omega_{T}^{2}-\omega^{2}-i \Gamma \omega}+\frac{\omega_{p}^{2}(\omega+i \gamma)}{\omega\left[\omega_{c}^{2}-(\omega+i \gamma)^{2}\right]}\right), \\
\epsilon_{2}(H) & =\frac{\epsilon_{\infty} \omega_{p}^{2} \omega_{c}}{\omega\left[(\omega+i \gamma)^{2}-\omega_{c}^{2}\right]}, \\
\epsilon_{3} & =\epsilon_{\infty}\left(1+\frac{\omega_{L}^{2}-\omega_{T}^{2}}{\omega_{T}^{2}-\omega^{2}-i \Gamma \omega}-\frac{\omega_{p}^{2}}{\omega(\omega+i \gamma)}\right) .
\end{aligned}
$$

Here, $\epsilon_{\infty}$ is the high-frequency dielectric constant, $\omega_{L}$ is the longitudinal optical phonon frequency, $\omega_{T}$ is the transverse optical phonon frequency, $\omega_{p}^{2}=n e^{2} /\left(m^{*} \epsilon_{0} \epsilon_{\infty}\right)$ defines the plasma frequency of free carriers of density $n$ and effective mass $m^{*}, \Gamma$ is the phonon damping constant, and $\gamma$ is the free-carrier damping constant. Finally, the magnetic field enters in these expressions via the cyclotron frequency $\omega_{c}=e H / m^{*}$. In what follows, we shall consider the following set of parameters taken from Ref. [35]: $\epsilon_{\infty}=15.7, \omega_{L}=3.62 \times 10^{13} \mathrm{rad} / \mathrm{s}$, $\omega_{T}=3.39 \times 10^{13} \mathrm{rad} / \mathrm{s}, \Gamma=5.65 \times 10^{11} \mathrm{rad} / \mathrm{s}, \gamma=$ $3.39 \times 10^{12} \mathrm{rad} / \mathrm{s}, n=1.07 \times 10^{17} \mathrm{~cm}^{-3}, \mathrm{~m}^{*} / \mathrm{m}=0.022$, and $\omega_{p}=3.14 \times 10^{13} \mathrm{rad} / \mathrm{s}$.

Let us start the discussion of the results by analyzing the effect of the magnitude of the magnetic field in the radiative heat transfer. In Fig. 2(a) we show the spectral conductance, defined as conductance per unit of photon energy, for two $\mathrm{InSb}$ particles of radius $250 \mathrm{~nm}$ and a gap of $500 \mathrm{~nm}$. The different curves correspond to different values of the magnetic field that is directed along the transport direction ( $z$-axis). These results were computed with the TDDA method of Ref. 30 discretizing each particle in 1791 cubic dipoles, which was checked to be enough to converge the results. As seen in Fig. 2(a), the spectral conductance in the absence of field is dominated by two peaks that, as we shall show below, are related to the localized plasmons of these particles. As the field increases, new peaks appear that disperse with the field following the magnetic-field-induced localized plasmons of these particles (see below). Notice that in some energy regions the magnetic field has a dramatic effect and it changes the spectral conductance by more than two orders of magnitude. Let us remark that the spectral conductance and the transmission coefficient, see Eq. (1), have basically the same energy dependence because at room temperature thermal factor $\partial \Theta(\omega, T) / \partial T$ is almost constant in the energy region of interest.

Let us now explore the role of the field direction. For this purpose, we show in Fig. 2(b) the dependence of the spectral conductance on the angle between the magnetic field and the transport direction for a fixed field 


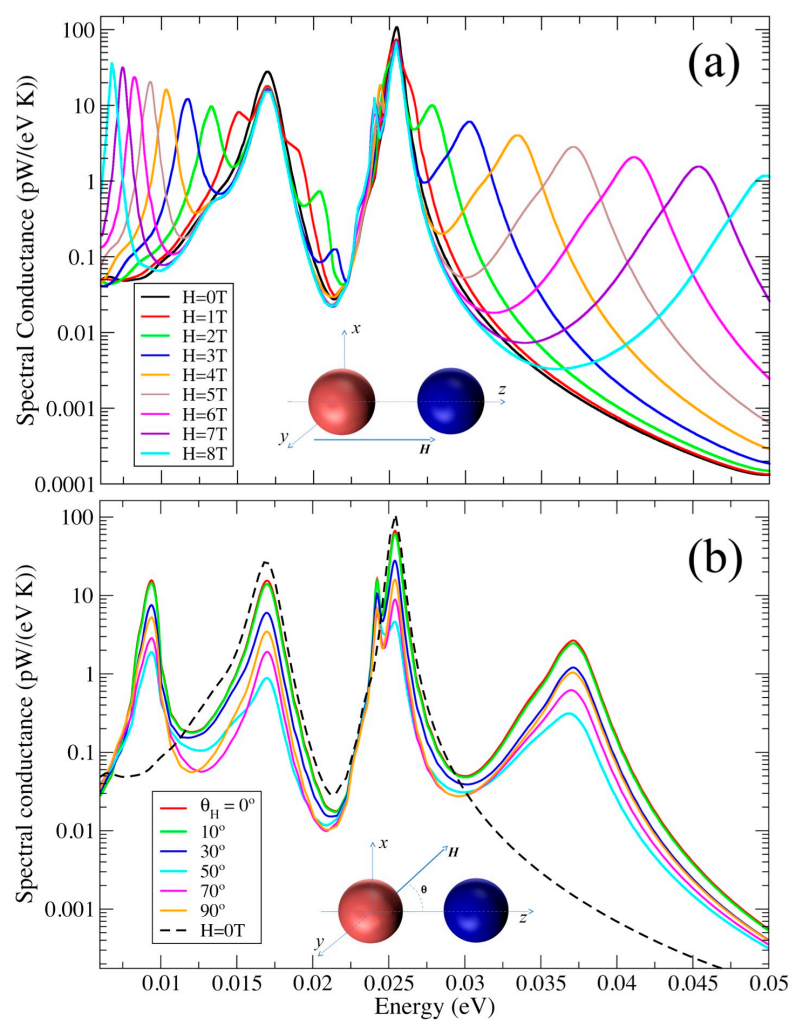

FIG. 2. (Color online) (a) Room temperature spectral conductance as a function of the photon energy for two InSb spheres of radius $250 \mathrm{~nm}$ separated by a gap of $500 \mathrm{~nm}$ for different values of the magnetic field point along the $z$-direction, see inset. (b) The corresponding spectral conductance for a magnetic field magnitude of $5 \mathrm{~T}$ and different angles between the magnetic field and the transport direction, see inset. The dashed line correspond to the case for zero field.

magnitude of $H=5 \mathrm{~T}$. As one can see, the rotation of the field strongly modulates the height of the spectral conductance peaks, but it does not change their position in energy. This modulation is the essence of the ATMR effect and originates from the magnetic-fieldinduced anisotropy in the photon tunneling. To quantify this effect we define the ATMR ratio $G(\theta) / G(\theta=0)$ between the conductance at a given angle $\theta$ and the conductance when the field points along the transport direction $(\theta=0)$. The numerical results obtained with TDDA for this ATMR ratio for the example of Fig. 2 are shown in Fig. 3(a) for different values of the field amplitude. As one can see, the conductance is strongly modulated by the field direction and it is symmetric around $\theta=90^{\circ}$. Notice also that, irrespective of the field value, the conductance is maximum at $\theta=0$. Moreover, for low fields the conductance reaches a minimum at $\theta=90^{\circ}$, while at higher fields (above $1 \mathrm{~T}$ ) the conductance exhibits two minima away from $90^{\circ}$. More importantly, the ATMR ratio reaches, e.g., a minimum of 0.51 for $H=1 \mathrm{~T}$ and of 0.127 for $H=5 \mathrm{~T}$. In terms of a thermal re-

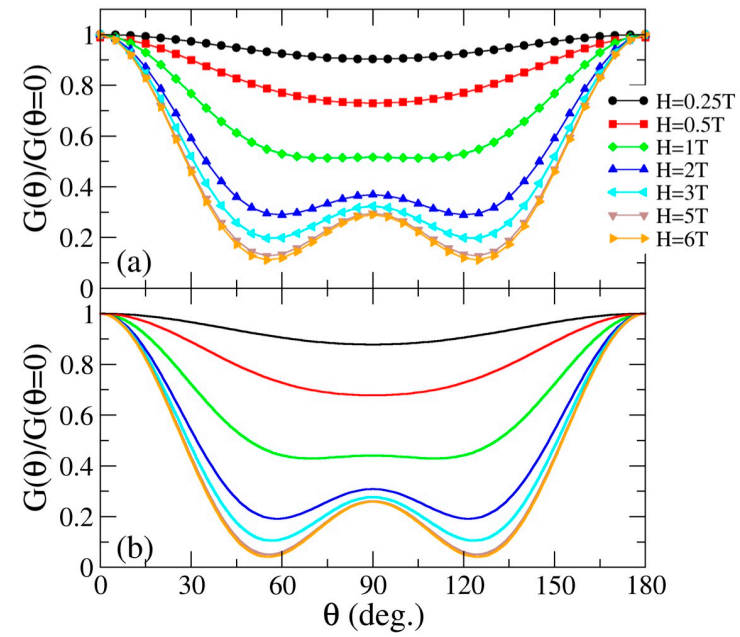

FIG. 3. (Color online) (a) Room-temperature thermal conductance as a function of the angle $\theta$ between the magnetic field and the transport direction for two InSb particles of radius $250 \mathrm{~nm}$, a gap of $500 \mathrm{~nm}$ and different values of the magnetic field magnitude. The conductance is normalized by the conductance at $\theta=0$. (b) The same as in panel (a), but calculated with the dipolar approximation.

sistance, $R=1 / G$, these ATMR ratios imply relative changes $[R(\theta)-R(\theta=0)] / R(\theta=0)$ of approximately $95 \%$ and $700 \%$, respectively, which are truly remarkable when we compare them with the $1 \%$ relative change in the resistance of spintronic devices for similar fields [4].

In order to understand these results, we now resort to the dipolar approximation in which each particle is considered to be a single dipole. Using Eqs. (115) we have computed the ATMR for the same parameters as in Fig. 3(a) and the results are shown in panel (b) of that figure. As one can see, the dipolar approximation qualitatively reproduces all the salient features discussed in the previous paragraph and this, in turn, justifies the use of this approximation to elucidate the underlying physics. As a next step, we want to understand the origin of the peaks in the spectral conductance, see Fig. 2(a). For this purpose, we investigate the electromagnetic modes supported by a single dipole. A convenient way to reveal the frequency and field dependence of these modes is to plot the total spectral emissivity of a single dipole, which in turn is equal to the total absorption cross section (its sum over the three spatial directions). This emissivity is given by [30] $\mathcal{E}(\omega, H)=(1 / 3) k_{0} \operatorname{Tr}\{\hat{\chi}\}$ and it is shown in Fig. 4 (a) as a function of the photon energy and the magnetic field amplitude for a particle radius of $250 \mathrm{~nm}$. Notice that the maxima of the emissivity nicely correspond to the peaks in the spectral conductance in Fig. 2(a). These maxima reveal the existence of two electromagnetic modes at zero field, which become up to six at finite field. In fact, from an analysis of the quasistatic polarizability, $\hat{\alpha}_{0}$, one can show that these resonant modes can 

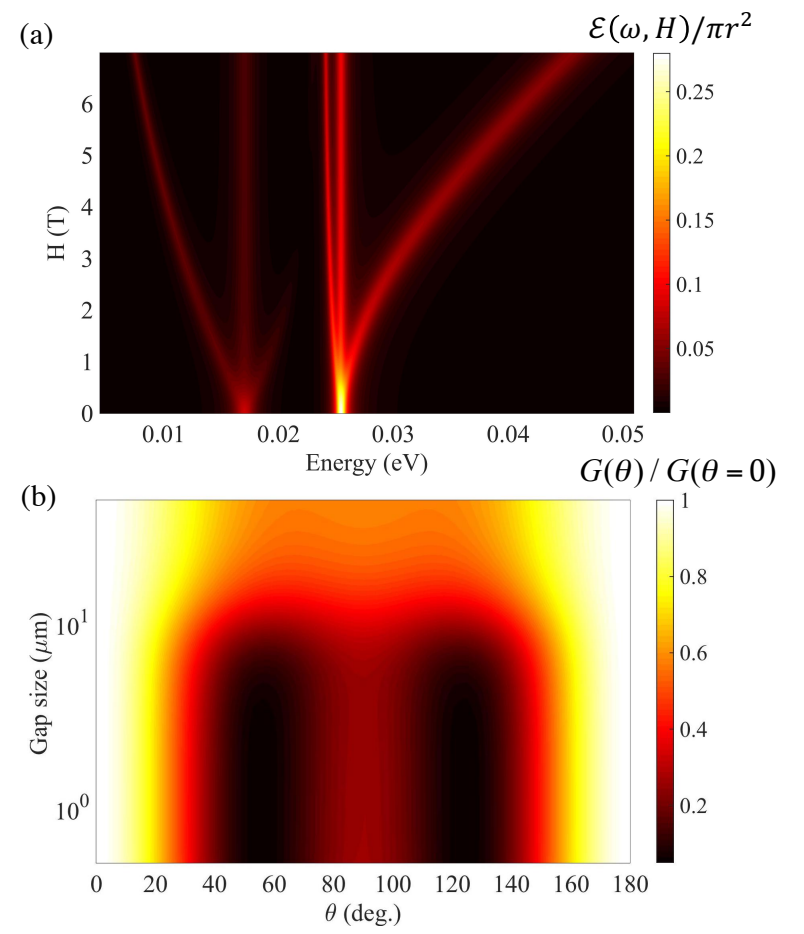

FIG. 4. (Color online) (a) Total spectral emissivity of a InSb dipole of radius $250 \mathrm{~nm}$ as a function of the photon energy and the magnitude of the magnetic field. The emissivity is normalized by the geometrical cross section $\pi r^{2}$. (b) Roomtemperature thermal conductance as a function of the angle $\theta$ between the magnetic field and the transport direction and as a function of the gap for two InSb spheres of radius 250 $\mathrm{nm}$ and a field magnitude $H=5 \mathrm{~T}$. The conductance is normalized by the conductance at $\theta=0$ for every gap and the calculations were done using the dipolar approximation.

be also obtained from the solutions of

$$
\operatorname{det}(\hat{\epsilon}(\omega, \boldsymbol{H})+2 \hat{1})=0=\left(\epsilon_{3}+2\right)\left[\left(\epsilon_{1}+2\right)^{2}-\epsilon_{2}^{2}\right] .
$$

At zero field, $\epsilon_{1}=\epsilon_{3}=\epsilon$ and $\epsilon_{2}=0$, and the previous condition reduces to the well-known condition for localized plasmons [36]: $\epsilon(\omega)=-2$. Notice that the condition of Eq. (8) is independent of $\theta$, as expected since we are dealing with an overall property of a spherical particle. This explains why the peak positions in the spectral conductance in Fig. 2(b) do not depend on the field direction.

Now, to understand the angular dependence of the ATMR, we use the fact that in the relevant frequency range the polarizability can be approximated by the quasistatic polarizability $\left(\hat{\alpha} \approx \hat{\alpha}_{0}\right)$. With this idea, and neglecting multiple scattering, i.e., using $\hat{C} \approx \hat{\mathcal{G}}$ in Eq. (4), it is straightforward to show that the ATMR ratio in the dipolar approximation adopts the form

$$
\frac{G(\theta)}{G(\theta=0)}=1+A \sin ^{2} \theta+B \sin ^{4} \theta
$$

where $A<0$ and $B>0$ (with $B<|A|$ ) are two coefficients that depend on the magnetic field amplitude and the gap. Moreover, one can show that at low fields (below $0.1 \mathrm{~T}$ ), the relative change in the conductance goes as $[G(\theta)-G(\theta=0)] / G(\theta=0) \propto-H^{2} \sin ^{2}(\theta)$, i.e., it is quadratic with the field. Equation $(9)$ describes very accurately the angular dependence shown in Fig. 3 . for both the exact results and those obtained with the dipolar approximation. This also explains why at low fields there is a single minimum at $\theta=90^{\circ}$ and two minima for higher fields. Physically, this angular dependence arises from the anisotropic thermal emission of these particles induced by the external magnetic field. This anisotropy leads in the near-field regime to the corresponding anisotropy in the photon tunneling.

The strong modulation of the ATMR is quite generic and it appears in a wide range of parameters. We illustrate this fact in Fig. 4(b) where we show the ATMR ratio computed within the dipolar approximation as a function of both $\theta$ and the gap size ranging from $500 \mathrm{~nm}$ to 50 $\mu \mathrm{m}$ for particles of radius $250 \mathrm{~nm}$. As one can see, the angular dependence of the ATMR ratio remains rather constant in the near-field regime, i.e., for gaps below 10 $\mu \mathrm{m}$, and its amplitude diminishes in the far-field regime. In all cases, this dependence is accurately described by Eq. (9).

Let us now discuss some potential applications of the ATMR effect. For small changes in the field direction, the heat conductance varies quadratically with the angle $\theta$, which shows that the heat flow can be efficiently modulated with a small variation of the magnetic field. The operational speed of such a modulation is only limited by the induction mechanisms used to tune the magnetic field so that the ATMR effect could be used to investigate heat transfer at few picoseconds scale. Thus, it could be used to investigate out-of-equilibrium heat transfer processes at the time scale of heat carrier relaxation. In addition, the ATMR effect could be implemented to make local measurements of temperature gradient as well as a thermal sensing of magnetic field orientation.

So in summary, we have predicted a thermal analogue of the AMR that is widely used in spintronics and proposed its use for an active control of near-field radiative heat transfer between MO objects. We have illustrated this effect with the case of two InSb particles and shown that the amplitude of the modulation of the magnetoresistance can be orders of magnitude larger than in electronic devices. This effect paves the way for an ultrafast thermal management with external magnetic fields.

We acknowledge funding from the Spanish MINECO (FIS2014-53488-P and MAT2014-58860-P) and the Comunidad de Madrid (S2013/MIT-2740). P.B.-A. acknowledges funding support from the Discovery Grant Program of CRSNG and J.C.C. thanks the DFG and SFB767 for sponsoring his stay at the University of Konstanz as Mercator Fellow. 
[1] W. Thomson, On the Electro-Dynamic Qualities of Metals: Effects of Magnetization on the Electric Conductivity of Nickel and of Iron, Proc. R. Soc. London 8, 546 (1857).

[2] I. Žutić, J. Fabian, and S. Das Sarma, Spintronics: Fundamentals and Applications, Rev. Mod. Phys. 76, 323 (2004).

[3] T. R. McGuire and R. I. Potter, Anisotropic Magnetoresistance in Ferromagnetic 3d Alloys, IEEE Trans. Magn. 11, 1018 (1975).

[4] R. C. O'Handley, Modern Magnetic Materials: Principles and Applications (Wiley, New York, 2000), p. 573.

[5] K. I. Bolotin, F. Kuemmeth, and D. C. Ralph, Anisotropic Magnetoresistance and Anisotropic Tunneling Magnetoresistance due to Quantum Interference in Ferromagnetic Metal Break Junctions, Phys. Rev. Lett. 97, 127202 (2006).

[6] M. Viret, M. Gabureac, F. Ott, C. Fermon, C. Barreteau, G. Autes, R. Guirardo-Lopez, Giant Anisotropic Magneto-Resistance in Ferromagnetic Atomic Contacts, Eur. Phys. J. B 51, 1 (2006).

[7] A. Sokolov, E. Y. Tsymbal, J. Redepenning, and B. Doudin, Quantized Magnetoresistance in Atomic-Size Contacts, Nat. Nanotechnol. 2, 171 (2007).

[8] F. Strigl, C. Espy, M. Bückle, E. Scheer, T. Pietsch, Emerging Magnetic Order in Platinum Atomic Contacts and Chains, Nat. Commun. 6, 6172 (2015).

[9] J. Schöneberg, F. Otte, N. Neél, A. Weismann, Y. Mokrousov, J. Kröger, R. Berndt, and S. Heinze, Ballistic Anisotropic Magnetoresistance of Single-Atom Contacts, Nano Lett. 16, 1450 (2016).

[10] D. Rakhmilevitch, S. Sarkar, O. Bitton, L. Kronik, O. Tal, Enhanced Magnetoresistance in Molecular Junctions by Geometrical Optimization of Spin-Selective Orbital Hybridization, Nano Lett. 16, 1741 (2016).

[11] D. Polder and M, Van Hove, Theory of Radiative Heat Transfer Between Closely Spaced Bodies, Phys. Rev. B 4, 3303 (1971).

[12] A. Kittel, W. Müller-Hirsch, J. Parisi, S.-A. Biehs, D. Reddig, and M. Holthaus, Near-field Heat Transfer in a Scanning Thermal Microscope, Phys. Rev. Lett. 95, 224301 (2005).

[13] E. Rousseau, A. Siria, G. Jourdan, S. Volz, F. Comin, J. Chevrier, and J.-J, Greffet, Radiative Heat Transfer at the Nanoscale, Nat. Photon. 3, 514 (2009).

[14] S. Shen, A. Narayanaswamy, and G. Chen, Surface Phonon Polaritons Mediated Energy Transfer between Nanoscale Gaps, Nano Lett. 9, 2909 (2009).

[15] R. S. Ottens, V. Quetschke, S. Wise, A. A. Alemi, R. Lundock, G. Mueller, D. H. Reitze, D. B. Tanner, and B. F. Whiting, Near-Field Radiative Heat Transfer between Macroscopic Planar Surfaces, Phys. Rev. Lett. 107, 014301 (2011).

[16] T. Kralik, P. Hanzelka, M. Zobac, V. Musilova, T. Fort, and M. Horak, Strong Near-Field Enhancement of Radiative Heat Transfer between Metallic Surfaces, Phys. Rev. Lett. 109, 224302 (2012).

[17] P. J. van Zwol, L. Ranno, and J. Chevrier, Tuning Near Field Radiative Heat Flux through Surface Excitations with a Metal Insulator Transition, Phys. Rev. Lett. 108, 234301 (2012).

[18] B. Song, Y. Ganjeh, S. Sadat, D. Thompson, A. Fior- ino, V. Fernández-Hurtado, J. Feist, F. J. Garcia-Vidal, J. C. Cuevas, P. Reddy, and E. Meyhofer, Enhancement of Near-Field Radiative Heat Transfer Using Polar Dielectric Thin Films, Nat. Nanotechnol. 10, 253 (2015).

[19] K. Kim, B. Song, V. Fernández-Hurtado, W. Lee, W. Jeong, L. Cui, D. Thompson, J. Feist, M. T. H. Reid, F. J. Garcia-Vidal, J. C. Cuevas, E. Meyhofer, and P. Reddy, Radiative Heat Transfer in the Extreme Near Field, Nature (London) 528, 387 (2015).

[20] R. St-Gelais, L. Zhu, S. H. Fan, and M. Lipson, NearField Radiative Heat Transfer between Parallel Structures in the Deep Subwavelength Regime, Nat. Nanotechnol. 11, 515 (2016).

[21] B. Song, D. Thompson, A. Fiorino, Y. Ganjeh, P. Reddy, E. Meyhofer, Radiative Heat Conductances between Dielectric and Metallic Parallel Plates with Nanoscale Gaps, Nat. Nanotechnol. 11, 509 (2016).

[22] M. P. Bernardi, D. Milovich, and M. Francoeur, Radiative Heat Transfer Exceeding the Blackbody Limit between Macroscale Planar Surfaces Separated by a Nanosize Vacuum Gap, Nat. Commun. 7, 12900 (2016).

[23] L. Cui, W. Jeong, V. Fernández-Hurtado, J. Feist, F.J. García-Vidal, J.C. Cuevas, E. Meyhofer, and P. Reddy, Study of Radiative Heat Transfer in Angström- and Nanometre-sized Gaps, Nat. Commun. 8, 14479 (2017).

[24] K. Kloppstech, N. Könne, S.-A. Biehs, A. W. Rodriguez, L. Worbes, D. Hellmann, and A. Kittel, Giant Heat Transfer in the Crossover Regime between Conduction and Radiation, Nat. Commun. 8, 14475 (2017).

[25] E. Moncada-Villa, V. Fernández-Hurtado, F. J. GarcíaVidal, A. García-Martín, and J. C. Cuevas, Magnetic Field Control of Near-Field Radiative Heat Transfer and the Realization of Highly Tunable Hyperbolic Thermal Emitters, Phys. Rev. B 92, 125418 (2015).

[26] P. Ben-Abdallah, Photon Thermal Hall Effect, Phys. Rev. Lett. 116, 084301 (2016).

[27] L. Zhu and S. Fan, Persistent Directional Current at Equilibrium in Nonreciprocal Many-Body Near Field Electromagnetic Heat Transfer, Phys. Rev. Lett. 117, 134303 (2016).

[28] I. Latella and P. Ben-Abdallah, Giant Thermal Magnetoresistance in Plasmonic Structures, Phys. Rev. Lett. 118, 173902 (2017).

[29] S. M. Rytov, Y. A. Kravtsov, and V. I. Tatarskii, Principles of Statistical Radiophysics Vol. 3 (Springer-Verlag, Heidelberg, 1989).

[30] R. M. Abraham Ekeroth, A. García-Martín, and J. C. Cuevas, Thermal Discrete Dipole Approximation for the Description of Thermal Emission and Radiative Heat Transfer of Magneto-Optical Systems, Phys. Rev. B 95 , 235428 (2017).

[31] B. T. Draine and P. J. Flatau, Discrete-Dipole Approximation for Scattering Calculations, J. Opt. Soc. Am. A 11, 1491 (1994).

[32] M. A. Yurkin and A. G. Hoekstra, The Discrete Dipole Approximation for Simulation of Light Scattering by Particles much Larger than the Wavelength, J. Quant. Spectrosc. Radiat. Transfer 106, 558 (2007).

[33] N. de Sousa, L. S. Froufe-Pérez, J. J. Sáenz, A. GarcíaMartín, Magneto-Optical Activity in High Index Dielectric Nanoantennas, Sci. Rep. 6, 30803 (2016). 
[34] L. Novotny and B. Hecht, Principles of Nano-Optics, (Cambridge University Press, Cambridge, 2012).

[35] E. D. Palik, R. Kaplan, R. W. Gammon, H. Kaplan, R. F. Wallis, and J. J. Quinn, Coupled Sur- face Magnetoplasmon-Optic-Phonon Polariton Modes on InSb, Phys. Rev. B 13, 2497 (1976).

[36] C. F. Bohren and D. R. Huffman, Absorption and Scattering of Light by Small Particles (Wiley, New York, 1998). 